Manuelle Medizin 2018 $56: 188$

https://doi.org/10.1007/s00337-018-0375-8

Online publiziert: 25 . Januar 2018

(c) Springer Medizin Verlag $\mathrm{GmbH}$, ein Teil von Springer Nature 2018

CrossMark

Joachim Hölle-Gindorf

Chirurgische Gemeinschaftspraxis Dres. med. M. Praeder/J. Hölle-Gindorf, Wittlich, Deutschland

\title{
Fasziendistorsionsmodell stellt keine Therapieoption bei Achillessehnenruptur dar
}

\section{Leserbrief zu \\ Fischer A, Fischer I (2017) Behandlung einer akuten Achillessehnenruptur mit Methoden des Fasziendistorsionsmodells. Manuelle Med 55:383-388. https://doi.org/10.1007/s00337- 017-0338-5}

Mit Interesse habe ich die Kasuistik der Kollegen Fischer gelesen. Sie propagieren die konservative Behandlung der akuten Achillessehnenruptur ohne jegliche Ruhigstellung nach dem Fasziendistorsionsmodell (FDM). In diesem Einzelfall mag die Behandlung erfolgreich gewesen sein. Man sollte sich aber keinesfalls dazu verleiten lassen, dieses Vorgehen weiterzuverbreiten. Warum? Zweifelsohne wurde hier eine Achillessehnenruptur korrekt mit den typischen Symptomen beschrieben. In Abb. 1, der Aufnahme aus der Magnetresonanztomographie 3 Tage nach dem Unfall, ist aber unschwer zu erkennen, dass die Rissstelle sehr hoch („, $8 \mathrm{~cm}$ oberhalb des unauffälligen Ansatzes am Calcaneus“) am Muskel-Sehnen-Übergang liegt. Dem operativ tätigen Kollegen ist bekannt, dass die Achillessehne auf dieser Höhe bereits deutlich schmaler wird und kontinuierlich in die Wadenmuskulatur übergeht. Die Achillessehne ist zwar gerissen, die benachbarten Muskelfasern sind laut zitiertem Radiologiebefund allerdings nur leicht ödematös aufgequollen. Dies erklärt auch, warum die Sehnenenden nicht auseinandergewichen sind. In diesem speziellen Fall wäre die Achillessehne sogar ohne jede Behandlung geheilt. In den meisten Fällen reißt die Achillessehne allerdings deutlich weiter distal. Die Sehnenenden sind aufgrund der hohen Muskelspannung dann deutlich retrahiert. Wenn sie sich in maximaler Plantarflexion des Fußes tatsächlich annähern lassen, ist eine konservative Behandlung (6 Wochen Ruhigstellung in Spitzfußstellung) durchaus möglich. Meine Erfahrung zeigt jedoch, dass die Sehne dann nicht selten nach Ende der Ruhigstellungsphase erneut reißt. Dies führt selbstredend $\mathrm{zu}$ erheblichen Frustrationen aufseiten der Patienten. Ich persönlich stelle meinen Patienten die verschiedenen Therapieoptionen - dazu gehört nicht die FDM! vor, bevorzuge aber eindeutig das operative Vorgehen. Das Ausheilungsergebnis ist nach operativer Adaptation der Sehnenenden i.d.R. sehr gut. Der Eingriff lässt sich problemlos in Lokalanästhesie durchführen und die Behandlungsdauer unterscheidet sich nicht von der des konservativen Vorgehens. Ich selbst würde mich im Falle einer Achillessehnenruptur auf jeden Fall operieren lassen. Vor der Veröffentlichung derart unkritischer Kasuistiken kann ich nur warnen, da diese sehr schnell verallgemeinert werden. Würde ich eine Achillessehnenruptur in meiner Praxis in der geschilderten Weise therapieren, würde ich mit an Sicherheit grenzender Wahrscheinlichkeit vor der ärztlichen Schiedsstelle landen und meine Haftpflichtversicherung würde mir den Vertrag kündigen.

\section{Korrespondenzadresse}

\section{Dr. med. J. Hölle-Gindorf}

Chirurgische Gemeinschaftspraxis Dres. med. M. Praeder/J. Hölle-Gindorf

Kurfürstenstraße 7A, 54516 Wittlich, Deutschland

hoe-gi@kabelmail.de

Interessenkonflikt. J. Hölle-Gindorf gibt an, dass kein Interessenkonflikt besteht. 